\title{
An Estimation Algorithm of Carrier Frequency Offset Based on Signal Reconstruction in Cooperative OFDM System
}

\author{
Jingjing Bao \\ Electronic Engineering Department \\ Dongguan Polytechnic College \\ Dongguan, China mainland \\ e-mail: 1936570746@qq.com
}

\author{
Yuan Liu \\ Electronic and Information College \\ South China University of Technology \\ Guangzhou, China Mainland \\ e-mail: eeyuanliu@gmail.com
}

\begin{abstract}
In cooperative OFDM system, because of the OFDM technology, cooperative communication system is very sensitive to carrier frequency offset, a tiny carrier frequency offset will cause the inter-carrier interference. An estimation algorithm of carrier frequency offset based on signal reconstruction is proposed in this paper, the value of carrier frequency offset is estimated by the tracking algorithm, and the estimation algorithm can adjust the action of system in real-time. Through the simulation, it indicated that the estimation algorithm proposed can track the change of carrier frequency offset correctly, and make appropriate adjustment quickly. Generally speaking, it has advantages of rapider response and more accurate estimation.
\end{abstract}

Keywords-Cooperative OFDM; Carrier Frequency Offset; Signal Reconstruction

\section{INTRODUCTION}

Cooperative OFDM system which introduces OFDM technology into cooperative communication, can not only improve spectrum utilization and resist frequency selective fading with the OFDM technology, but also advance ability to resist multi-path fading with cooperative technology. It has advantages of OFDM technology and cooperative technology, besides removes disadvantages of them, will be a promising solution in the future communication field.

OFDM is very sensitive to carrier frequency offset (CFO), carrier frequency synchronization becomes a question which must be considered seriously in practice, and it will be more complex in Cooperative OFDM system. Some researches on carrier frequency synchronization in Cooperative OFDM system have been done. Frequency synchronization is realized in [1], which exploits feedback signals to adjust frequency adaptively. The problem of multicarrier frequency offset (M-CFO) is solved in [2], which employs decision feedback equalization algorithm based on minimum mean-squared error. Frequency and phase can be tracked and locked by phase locked loop (PLL), which is proposed in [3], however, it is extremely difficult to lock frequency and phase at high rate for much time PLL required, which can easily cause change of carrier ahead of tracking of PLL greatly. After analyzing structure of received signal, the interference in received signal is eliminated in [4] by means of iteration and successive approximation. A frame preamble symbol with fine autocorrelation and cross-correlation is constructed in [5] to estimate frequency and timing offset, ultimately to realize synchronization. The CFO in spacefrequency coding cooperative system is studied in [6][7], then a M-CFO balanced detection algorithm is proposed. According to OFDM structure, a specific space-frequency coding and the corresponding inter-carrier inference cancellation algorithm is designed in [8], which solves the $\mathrm{M}-\mathrm{CFO}$ in cooperative system. The preamble symbol is used to estimate CFO in [9], and the result that estimation error is reduced with the increase of SNR is pleasing, but the estimation is only applicable at transmission stage. A blind carrier synchronization scheme based on signal reconstruction in OFDM system is proposed in [10], which tracks CFO and compensates it according to MMSE.

After being inspired by blind carrier synchronization scheme based on signal reconstruction in OFDM system in [10], the problem in [9] that the preamble symbol can only estimate the frequency offset at transmission stage but can not track change of frequency offset is analyzed again, and eventually the carrier synchronization scheme based on signal reconstruction in cooperative OFDM system is proposed in this paper to solve the problem in [9].

\section{SySTEM MODEL AND PROBLEM FORMULATION}

To simplify, we assume that there are only three nodes, that is the source node $(\mathrm{S})$, the relay node $(\mathrm{R})$ and the destination node (D) in cooperative OFDM system as shown in Fig.1. The carrier synchronous scheme based on signal reconstruction is divided into two stages as shown in Fig.2: $\mathrm{S}$ and $\mathrm{R}$ transmit data alternately at a half speed in the frequency offset tracking stage, while in the monitoring stage they transmit together at a maximum speed.

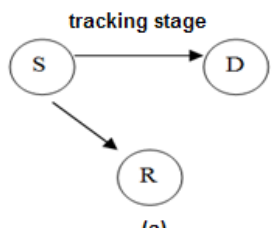

(a)

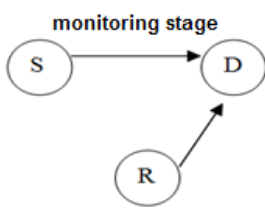

(b)
Figure 1. Model of cooperative OFDM system 


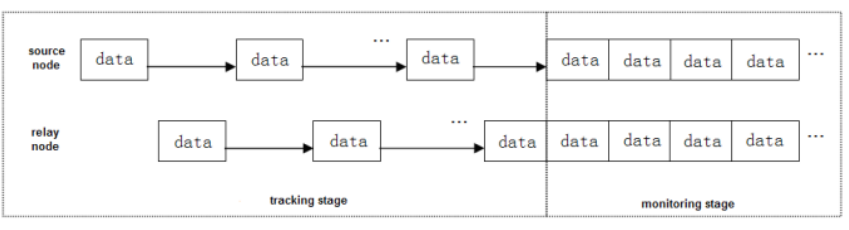

Figure 2. Diagram of carrier synchronization scheme based on signal reconstruction

A set of data to be transmitted is denoted as $X(0)$, $X(1), \ldots, X(N-1)$, they are converted into discrete OFDM symbols in time domain by IFFT transformation, which can be expressed as the formula below

$$
S(n)=\sum_{k=0}^{N-1} X(k) e^{j 2 \pi n k / N}(n=0,1, \ldots, N-1)
$$

If the channel discrete pulse response is $h(n)$, the normalized CFO is $\varepsilon_{0}$, the cyclic prefix (CP) is added into $s(n)$ to remove the inter-symbol-interference (ISI), OFDM symbols at receiver can be expressed as the formula below

$$
r\left(n, \varepsilon_{0}\right)=(s \otimes h)(n) \mathrm{e}^{\mathrm{j} 2 \pi \varepsilon_{0}(n+L) / N}+Z(n)(n=0,1, \ldots, N-1)
$$

In the formula, $L$ is the length of $\mathrm{CP}, Z(n)$ is the channel noise at sampling time $n$, which complies with Gaussian distribution, $\otimes$ is the circular convolution.

$r\left(n, \varepsilon_{0}\right)$ is transformed into $R\left(k, \varepsilon_{0}\right)$ by IFFT, the estimated value of $X(k)$ is $\tilde{X}(k)$ with maximum likelihood judgment in absence of channel interference. Generally, frequency offset and additive noise can be removed after maximum likelihood judgment, so $X(k)$ is approximately equal to $\tilde{X}(k) . \tilde{X}(k)$ can be reconstructed by $\tilde{r}(n)$

$$
\begin{aligned}
\tilde{r}(n, \varepsilon)=\operatorname{IFFT}[\tilde{X}(k) \hat{H}(k)] e^{j 2 \pi x(n+L) / N} & =\tilde{r}(n) e^{j 2 \pi \varepsilon(n+L) / N} \\
(n & =0,1, \ldots, N-1)
\end{aligned}
$$

In the formula, $\hat{H}(k)$ is the estimated value of channel, $\varepsilon$ is the estimated value of $\varepsilon_{0}$. If $\varepsilon=\varepsilon_{0}$, the values of $r\left(n, \varepsilon_{0}\right)$ and $\tilde{r}(n, \varepsilon)$ are nearly equal, the computation of estimated value of $\varepsilon_{0}$ is transformed into the computation of minimum of $\varepsilon$ in formula (4)

$$
\min _{\varepsilon} \phi(n, \varepsilon)(n=0,1, \ldots, N-1)
$$

In the formula, $\phi(n, \varepsilon)=\left|r\left(n, \varepsilon_{0}\right)-r(n, \varepsilon)\right|^{2}$. If $r\left(n, \varepsilon_{0}\right)=a(n)+b(n)$, $\tilde{r}(n, \varepsilon)=\tilde{a}(n)+j \tilde{b}(n), \quad$ so

$$
\phi(n, \varepsilon)=\left|a(n)+j b(n)-[\tilde{a}(n)+j \tilde{b}(n)] e^{j 2 \pi \varepsilon(n+L) / N}\right|^{2}
$$

When $\varepsilon \ll 1$, we expand exponential part of formula (5) by Taylor formula, then retain linear part of result, so (5) can be approximately expressed as

$\phi(n, \varepsilon) \approx$

$$
[a(n)-\tilde{a}(n)+2 \pi \tilde{b}(n) \varepsilon n / N]^{2}+[b(n)-\tilde{b}(n)-2 \pi \tilde{a}(n) \varepsilon(n+L) / N]^{2}
$$

Compute derivative of $\phi(n, \varepsilon)$ and let $\frac{d \phi(n, \varepsilon)}{d \varepsilon}=0$, so

$$
\varepsilon(n)=\frac{\tilde{a}(n) b(n)-a(n) \tilde{b}(n)}{\left[\tilde{a}^{2}(n)+\tilde{b}^{2}(n)\right] 2 \pi(n+L) / N}(n=0,1, \ldots, N-1)
$$

Because $r\left(n, \varepsilon_{0}\right)$ and $\tilde{r}(n, \varepsilon)$ are both random variables that are influenced by additive noise, $\varepsilon(n)$ will be an unstable estimated value, to eliminate instability of $\varepsilon(n)$, let

$$
\hat{\varepsilon}=\frac{\sum_{n=0}^{N-1}[\tilde{a}(n) b(n)-a(n) \tilde{b}(n)]}{\sum_{n=0}^{N-1}\left[\left(\tilde{a}^{2}(n)+\tilde{b}^{2}(n)\right) 2 \pi(n+L) / N\right]}
$$

We can propose carrier frequency tracking algorithm of receiver according to estimated result of frequency offset in formula (8), then construct carrier tracking loop to correct frequency offset of oscillator at receiver.

\section{Proposed Estimation Algorithm OF CFO}

The proposed carrier synchronous scheme based on signal reconstruction can be specifically divided into two stages: the tracking stage and the monitoring stage. In the tracking stage, $\mathrm{S}$ and $\mathrm{R}$ transmit data to $\mathrm{D}$ alternately, D estimates values of CFO of $S$ and $R$ with the method used in [10], then sends results back to $S$ and $R$ to help them adjust their carrier frequency; when the estimated value of frequency offset is below to a certain threshold, the system will transfer into the monitoring stage, $\mathrm{S}$ and $\mathrm{R}$ transmit data at the maximum speed, D monitors frequency offset continually, the monitored value of the current frame can compensate the next one; when the monitored value of frequency offset is above to a certain threshold, the system will reverse to the tracking stage.

The tracking algorithm at the tracking stage:

i. Let the initial normalized CFO of $\mathrm{S}$ and $\mathrm{R}$ are $\varepsilon_{1}$ and $\varepsilon_{2}$ respectively, the frame label is $n$;

ii. Compute the frequency offset estimated values of $S$ and

$\mathrm{R}$, which are expressed as $\hat{\varepsilon}_{1}$ and $\hat{\varepsilon}_{2}$;

iii. Feed the estimated values $\hat{\varepsilon}_{1}$ and $\hat{\varepsilon}_{2}$ back to $\mathrm{S}$ and R; iv. Set $\varepsilon_{1}=\varepsilon_{1}-\hat{\varepsilon}_{1}$ and $\varepsilon_{2}=\varepsilon_{2}-\hat{\varepsilon}_{2}$ to rectify the frequency offset; v. Let $n=n+1$, then go to ii.

When $\hat{\varepsilon}_{1}$ and $\hat{\varepsilon}_{2}$ are both below to a certain threshold, the system will transfer into the monitoring stage, $\mathrm{S}$ and $\mathrm{R}$ transmit data at the maximum speed. It is necessary to set the threshold value ahead of the system transferring into the monitoring stage from the tracking stage.

At the monitoring stage, let the remained CFO of S and $\mathrm{R}$ are $\Delta \varepsilon_{1}$ and $\Delta \varepsilon_{2}$ after the tracking stage. If the data to be 
transmitted can be expressed as $X(0), X(1), \ldots, X(N-1)$, then the data at the receiver will be

$r\left(n, \Delta \varepsilon_{1}, \Delta \varepsilon_{2}\right)=\left(s_{1} \otimes h_{1}\right)(n) \mathrm{e}^{j 2 \pi \varepsilon_{1}(n+L) / N}+\left(s_{2} \otimes h_{2}\right)(n) \mathrm{e}^{j 2 \pi \Delta \varepsilon_{2}(n+L) / N}+Z(n)$

Define $s_{1}$ and $s_{2}$ as discrete signals in time domain of $X(0), X(1), \ldots, \quad X(N-1)$ at $\mathrm{S}$ and $\mathrm{R}$ which is achieved by IFFT, when $\mathrm{S}$ and $\mathrm{R}$ transmit the same data, that is, $s_{1}=s_{2}=s$, the data which is named as $r\left(n, \Delta \varepsilon_{1}, \Delta \varepsilon_{2}\right)$ at the receiver can be expressed as formula (10)

$r\left(n, \Delta \varepsilon_{1}, \Delta \varepsilon_{2}\right)=\operatorname{IFFT}\left(S H_{1}\right) \mathrm{e}^{j 2 \pi \varepsilon_{1}(n+L) / N}+\operatorname{IFFT}\left(S H_{2}\right) \mathrm{e}^{j 2 \pi \varepsilon_{2}(n+L) / N}+Z(n)$

It can be indicated from [10] that the errors can be controlled within $1 \%$ by the tracking of frequency offset, and the number will be less when the signal to the noise ratio is high enough, that is, $\Delta \varepsilon_{1} \leq \pm 0.1$ and $\Delta \varepsilon_{2} \leq \pm 0.1$ after the tracking stage. So

$r\left(n, \Delta \varepsilon_{1}, \Delta \varepsilon_{2}\right) \approx$

$\operatorname{IFFT}\left(\mathrm{SH}_{1}\right)\left(1+j 2 \pi \Delta \varepsilon_{1}(n+L) / N\right)+\operatorname{IFFT}\left(\mathrm{SH}_{2}\right)\left(1+j 2 \pi \Delta \varepsilon_{2}(n+L) / N\right)+Z(n)$

$\approx \operatorname{IFFT}\left(S\left(H_{1}+H_{2}\right)\right) e^{j 2 \pi \Delta \varepsilon_{1}(n+L) / N}+\tilde{Z}(n)$

From formula (10), $r\left(n, \Delta \varepsilon_{1}, \Delta \varepsilon_{2}\right)$ can be considered as the result that $s(n)$ passed through the channel whose frequency domain response is $H_{1}+H_{2}$, the receiver estimates frequency offset with formula (8), then takes the estimated value as the monitoring value to adjust CFO, if the estimated value is above to a certain threshold, the system will be back to the $\mathrm{t}$ racking stage.

\section{SimUlation RESUltS AND ANALYSIS}

Figure 3 and Figure 4 show the performance of the estimation algorithm of CFO based on signal reconstruction. Figure 3 is simulated when there is no frequency offset at $\mathrm{S}$ and $\mathrm{R}$, the remained frequency offset errors are $1 \%$ and $0.5 \%$ respectively after the tracking stage. Figure 4 is simulated when carrier frequency of the current frame is shifted by $0.5 \%$ relative to that of the former frame, the remained frequency offset errors are $1 \%$ and $0.5 \%$ respectively after the tracking stage. The modulation of OFDM system is 16QAM, the number of carriers is 256 , the length of $\mathrm{CP}$ is 32 , $\mathrm{SNR}=20 \mathrm{~dB}$. To simplify channel estimation algorithm, we select 5-path channel, the impulse responses of it in time

domain are $h(0)=1+j \quad, \quad h(7)=0.5-0.3 j \quad, \quad h(9)=0.3+0.2 j \quad$, $h(21)=0.2-0.1 j, \quad h(30)=0, h(n)=0(n \neq 0,7,9,21,30)$.

From figure 3 , it is showed that when there are two different remained frequency offsets, the detected value for frequency offset of the composite signal which is detected at the monitoring stage will be between them. The simulation result demonstrates that the synchronous scheme of carrier frequency cannot eliminate the remained $\mathrm{CFO}$ of $\mathrm{S}$ and $\mathrm{R}$ perfectly at the monitoring stage, but it can decrease frequency offset value of the remained carrier effectively.

It is compared the different remained frequency offset with the same one in figure 4 when there is CFO at the monitoring stage. The simulation result demonstrates that in this case the synchronous scheme of carrier frequency can decrease the remained $\mathrm{CFO}$ of $\mathrm{S}$ and $\mathrm{R}$ effectively, even control it within $1 \%$.

From the above analysis it is concluded that the estimation algorithm of CFO based on signal reconstruction can track the change of $\mathrm{CFO}$ correctly and makes the corresponding adjustment in time.
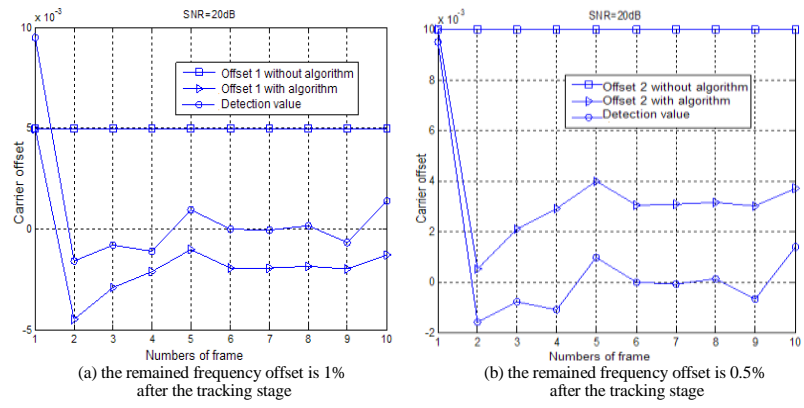

Figure 3. Diagram of the remained frequency offset errors at the monitoring stage when there is no $\mathrm{CFO}$ at $\mathrm{S}$ and $\mathrm{R}$

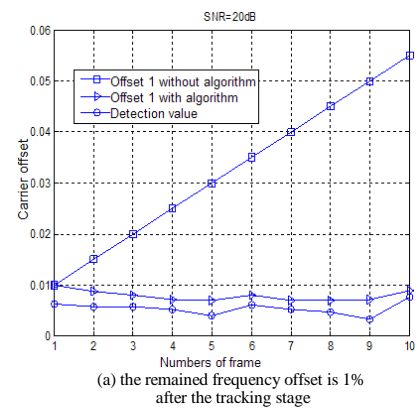

Figure 4. Diagram of the remained frequency offset errors at the monitoring stage when there is $\mathrm{CFO}$ at $\mathrm{S}$ and $\mathrm{R}$

\section{CONCLUSION}

An estimation algorithm of $\mathrm{CFO}$ based on signal reconstruction in cooperative OFDM system is proposed in this paper. It solves the problem in [9] that the estimation algorithm of CFO based on preamble symbols is lack of tracking the change of CFO. The inspiration of innovative idea in this paper is benefit from [10], which presents an estimation algorithm of $\mathrm{CFO}$ based on signal reconstruction 
in OFDM system. The simulation result demonstrates that the proposed algorithm in this paper can track the change of CFO correctly and make the corresponding adjustment in time. It not only responses faster, but also is higher in estimation accuracy.

\section{REFERENCES}

[1] P.A.Parker, D.W.Bliss, P.Mitran, and V.Tarokh, "Adaptive Frequency Synchronization for Collaborative Communication Systems," 27th International Conference on Distributed Computing Systems Workshops, pp. 82-85, 2007.

[2] D. Veronesi, and D. L. Goeckel, "Multiple frequency offset compensation in cooperative wireless systems," IEEE Global Telecommun Conference, pp. 1-5.2006.

[3] D.R. III Brown, G.B. Prince, and J.A. McNeill, “A method for carrier frequency and phase synchronization of two autonomous cooperative transmitters," IEEE 6th Workshop on Signal Processing Advances in Wireless Communications, pp. 260-264, 2005.

[4] D.Sreedhar, and A.Chockalingam, "ICI-ISI Mitigation in Cooperative SFBC-OFDM with Carrier Frequency Offset," IEEE 18th International Symposium on Personal, Indoor and Mobile Radio Communications, pp. 1-5, 2007.
[5] Oh-Soon Shin, A.M.Chan, H.T. Kung, and V. Tarokh, "Design of an OFDM Cooperative Space-Time Diversity System," Vehicular Technology, IEEE Transactions on, vol:56(4), pp. 2203-2215, 2007.

[6] F. Tian, X.G. Xia, and P. C. Ching, "Signal detection for space frequency coded cooperative communication system with multiple carrier frequency offsets," IEEE Wireless Communications Networking Conference (WCNC), pp. 1221-1225, 2007.

[7] F. Tian, X.G. Xia, and P. C. Ching, "Equalization in Space Frequency Coded Cooperative Communication System with Multiple Frequency Offsets," International Symposium on Signals, Circuits and Systems, vol:2(1), pp. 13-14, 2007.

[8] Huiming Wang, Xiang-Gen Xia, and Qinye Yin, "Distributed spacefrequency codes for cooperative communication systems with multiple carrier frequency offsets," IEEE Transactions on Wireless Communications, vol:8(2), pp. 1045-1055, 2009.

[9] Jingjing Bao, and Xinghua Zhao, "Improved estimation algorithm of carrier offset based on the preamble signal in OFDM cooperative system," Video Engineering, vol:20(2), pp. 12-15, 2013.

[10] Yan Du, and Xiaomin Zhang, "Estimation algorithm of carrier offset based on signal reconstruction in OFDM system," Journal of Shandong University(engineering), vol:33(1), pp. 5-8, 2003. 